\title{
Medicago polymorpha-mediated antibacterial silver nanoparticles in the reduction of methyl orange
}

https://doi.org/10.1515/gps-2018-0030

Received January 22, 2018; accepted April 24, 2018; previously published online June 18, 2018

Abstract: The aim of the presented work was to assess the potential of Medicago polymorpha extract to synthesize silver nanoparticles (AgNPs) as a green method. It was a simple one-step synthesis approach and the product obtained was characterized by UV-visible spectroscopy, Fourier transform infrared (FTIR), powder X-ray diffraction, thermogravimetric analysis, and field-emission scanning electron microscopy (FE-SEM). At room temperature, the optimum time for the completion of the reaction (i.e. the formation colloidal solution) was just 5 min. FE-SEM images showed that AgNPs were predominantly in spheres, whereas FTIR spectrum analysis inferred that gallic acid present in the extract initially reduced silver ions to elemental silver. The carboxylic and hydroxyl groups of biomolecules present in the extract stabilized AgNPs by passivating the surface to prevent aggregation, resulting in uniform distribution. The antibacterial activity of synthesized AgNPs showed effective inhibitory effects against waterborne pathogens, including Escherichia coli (E. coli) and Staphylococcus aureus (S. aureus), at a minimum inhibitory concentration of $10 \mu \mathrm{g} / \mathrm{ml}$. Membrane permeability and respiration studies were also performed to assess the surface role of the synthesized AgNPs. The prepared AgNPs exhibited excellent antioxidant activity and catalytic reduction of methyl orange with a rate constant of $6.8 \times 10^{-3} \mathrm{~s}^{-1}$.

Keywords: biogenic silver nanoparticles; dye removal; membrane permeability.

\footnotetext{
*Corresponding author: Sher Bahadar Khan, Center of Excellence for Advanced Materials Research and Chemistry Department, King Abdulaziz University, P.O. Box 80203, Jeddah 21589, Saudi Arabia, e-mail: sbkhan@kau.edu.sa

Muhammad Ismail, Saima Gul, M.I. Khan and Murad Ali Khan: Department of Chemistry, Kohat University of Science and Technology Kohat-26000, Khyber Pakhtunkhwa, Pakistan Abdullah M. Asiri: Center of Excellence for Advanced Materials Research and Chemistry Department, King Abdulaziz University, P.O. Box 80203, Jeddah 21589, Saudi Arabia
}

Ә Open Access. (cc)BY $\odot 2019$ Walter de Gruyter GmbH, Berlin/Boston

\section{Introduction}

During the last two decades, researchers have started taking a considerable interest in the area of nanoparticle synthesis. Metallic nanoparticles exhibit a wide range of interesting applications, such as catalytic, optical, electrical, biological labeling, and magnetic properties [1]. Research on nanoscale metals is inevitable due not only to the variety of applications in diverse fields but also to different synthetic approaches [2]. Chemical and physical syntheses of metal nanoparticles have considerable environmental problems, both laborious and economically expensive [3]. However, biological methods have new, simple, and ecofriendly green chemical approaches toward the synthesis of metallic nanoparticles [4]. Green synthetic approaches toward metallic nanoparticles proved to be better than chemical and physical methods in respect that these are environment friendly, cost effective, and easily synthesized on a large scale and do not need high temperatures, pressure, or toxic chemicals $[5,6]$. Among different metallic nanoparticles, silver nanoparticles (AgNPs) have attracted extensive attention in the fields of biology and therapeutics viz. antimicrobials, antifungal, antimalarial, and bimolecular detection [7]. AgNPs have promising potential in the field of nanomedicine because of their unique activity against Gram-negative and Gram-positive bacteria, protozoa, fungi, and viruses and tumoricidal effect against different cell lines [8-10]. Pathogenic bacteria have developed drug resistance against several antibiotics; for example, Candida albicans is resistant to fluconazole and Staphylococcus aureus has developed resistance to methicillin. Salmonella typhi has exhibited resistance to a variety of drugs, including ampicillin, chloramphenicol, quinolones, and trimethoprim. Similarly, Escherichia coli also showed resistance to a variety of antibiotics such as kanamycin, ampicillin, sulfisoxazole, streptomycin, tetracycline, and ticarcillin; this situation is alarming and poses a major challenge for researchers. The use of AgNPs plays an important role to combat drug-resistant pathogenic microorganisms. 
Silver and its compounds have germicidal effects and are effective against lower organisms with no harm to higher animals [11].

Dyes are persistent molecules that show resistance to biodegradability, and pollution caused by dye waste is increasing alarmingly each passing day. Dyes and their color removal have currently become a major scientific interest $[12,13]$. Methyl orange (MO) belongs to a group of organic azo dyes, which is toxic in nature and most widely used in foodstuff, textile, leather, and paper industries. Since ancient times, it has been in use as a common dye in the textile industry, and its discharge in our ecosystem is dangerously disturbing, which has a direct impact on human health and is a great threat to animals and aquatic life. It has been widely used as a $\mathrm{pH}$ indicator in research laboratories and generally discharged without further treatment. MO dye is nonbiodegradable due to complex aromatic structure and xenobiotic properties [14].

Medicago genus is fed crops with good digestibility, protein content, phenolic content, flavonoid and isoflavonoid contents, and other nutrients; the leaves contain proteins and carotenoid contents in high amounts, making it suitable for feeding into monogastric such as poultry and cattle as well as a key ingredient in high protein human health supplements [15].

Looking at the interesting role of Medicago polymorpha (M. polymorpha), in the present study, AgNPs are synthesized using $M$. polymorpha leaf extract, which is an unprecedented green synthesis route of AgNPs and their characterization. The synthesized material was used to investigate the 1-1-diphenyl-2-picrylhydrazyl (DPPH) free radical scavenging activity (RSA) and antibacterial activity against waterborne bacteria $E$. coli and S. aureus. Further, the synthesized AgNPs were used as a catalyst for the reduction of the water-soluble azo dye MO, which is a serious threat to aquatic ecosystems.

\section{Materials and methods}

\subsection{Materials}

Silver nitrate $\left(\mathrm{AgNO}_{3}\right)$ was purchased from Merck (Germany). MO and sodium borohydride $\left(\mathrm{NaBH}_{4}\right)$ were from BDH Laboratory Supplies (England). Nutrient agar was from Oxoid Ltd. (England). Ascorbic acid and DPPH were from Sigma-Aldrich (Germany). Distilled deionized water was used throughout the reactions. Aluminum foil was used for the wrapping of reaction tubes and all reactions were carried out in triplicate.

\subsection{Plant material and extract preparation}

Fresh leaves of M. polymorpha were collected and used for the preparation of the extract. For extract preparation, $25 \mathrm{~g}$ leaf were weighed and thoroughly washed with double-distilled water, dried, chopped into fine pieces, and finally boiled for $25 \mathrm{~min}$ at $70^{\circ} \mathrm{C}$ in $100 \mathrm{ml}$ deionized water. The extract was filtered through filter paper (pore size: 110 $\mu \mathrm{m}$ ) followed by another filtration through filter paper (pore size: 90 $\mu \mathrm{m})$. The filtrate was stored in a freezer at $-4^{\circ} \mathrm{C}$ for further use.

\subsection{Synthesis of AgNPs}

Aqueous $\mathrm{AgNO}_{3}$ solution in deionized water was used to synthesize AgNPs. The M. polymorpha leaf extract $(15 \mathrm{ml}$ ) was added to $85 \mathrm{ml}$ of $3 \mathrm{~mm}$ aqueous solution of $\mathrm{AgNO}_{3}$ to obtain AgNPs. The mixture was kept at room temperature for $5 \mathrm{~min}$, and immediately observed the color changed from light yellow to dark brown. Particles were collected and the unreacted biomass residue was removed by five times centrifugation at 6000 revolutions per minute (rpm) for $10 \mathrm{~min}$. The resulting AgNP pellet was washed with double-distilled water followed by distilled ethanol and finally with acetone and dried at room temperature. The nanoparticles thus obtained looked black in color.

\subsection{In vitro antibacterial activity against waterborne bacteria}

The minimum inhibitory concentrations (MICs) for M. polymorpha extract, $\mathrm{AgNO}_{3}$, and AgNPs were determined by the MTT assay using 96-well microtiter plates against $E$. coli and $S$. aureus by the standard literature method [16]. ELISA reader (EMax precision microplate reader) was used to record the mean of live cells. Based on varying concentrations of the tested material, MIC was determined when there was no increase in the $\mathrm{OD}_{595}$ and was zero.

\subsection{Changes in membrane permeability of bacterial cells}

Viable bacterial cultures of $E$. coli and S. aureus in nutrient broth were treated with the tested material to study the membrane permeability. Then, $10 \mathrm{ml}$ cultures were centrifuged at $6000 \mathrm{rpm}$ for $10 \mathrm{~min}$ and the pellet obtained was suspended in sterile water. About $5 \mathrm{ml}$ of the suspension were exposed to $100 \mathrm{ppb}$ AgNPs and the conductance was recorded after incubation for 1, 3, 6, and $24 \mathrm{~h}$ using a Jenway conductivity meter (3540 Bench combined conductivity/pH meter) (England).

\subsection{Free radical quenching assay}

The antioxidant property of AgNPs was evaluated by monitoring the capability of quenching DPPH free radical into a nonradical form. A reaction mixture containing $2 \mathrm{ml}$ of $50 \mu \mathrm{M}$ DPPH and $2 \mathrm{ml}$ of the M. polymorpha extract synthesized AgNP solution. Varying concentrations of nanoparticles used were about 10,50 , and $100 \mu \mathrm{g} / \mathrm{ml}$. The reaction was monitored and incubated at $37^{\circ} \mathrm{C}$ in the dark for $40 \mathrm{~min}$ 
and the change in color was measured at $517 \mathrm{~nm}$ using UV-visible (UV-vis) spectrophotometer. Ascorbic acid of the same concentrations $(10,50$, and $100 \mu \mathrm{g} / \mathrm{ml})$ was used as a positive control. Here, we used $50 \mu \mathrm{M}$ concentrations of DPPH in consonance with the accuracy in the measurements of the spectrophotometer. A higher percentage of scavenging activity indicated lower absorbance of the reaction mixture. The antioxidant activity or RSA was calculated in percent inhibition using the following Equation (1) [17]:

$$
\% \mathrm{RSA}=\left(A_{\mathrm{DPPH}}-A_{\text {solution }}\right) / A_{\mathrm{DPPH}} \times 100
$$

where $A_{\mathrm{DPPH}}$ and $A_{\text {solution }}$ are the absorption of the pure DPPH solution and after treatment with AgNPs, respectively.

\subsection{Photocatalytic reduction of MO}

The prepared nanoparticles were evaluated for the catalytic reduction of MO dye by sodium borohydride $\left(\mathrm{NaBH}_{4}\right)$. For the reduction, $0.02 \mathrm{~mm}$ concentration of MO dye was used. Quartz cuvette was used as a reaction vessel. About $2.5 \mathrm{ml}$ of the MO solution were introduced into a UV cuvette having $0.5 \mathrm{ml} \mathrm{NaBH}_{4}$ solution and its UV-vis spectra were recorded. For the reduction of MO, $5 \mathrm{mg}$ AgNP catalyst was introduced into the reaction mixture. The reduction of MO was initiated after the addition of AgNP catalyst to the UV cuvette. The absorption spectrum of the reaction mixture was recorded continuously with an interval of $30 \mathrm{~s}$ and the absorbance value was monitored using a UV-vis spectrophotometer (UV-1800, Shimadzu, Japan) and the $\lambda_{\max }$ values were compared.

\subsection{Instrumentation}

2.8.1 UV-vis spectroscopy: The catalytic reduction of dye and the formation of nanoparticles were confirmed using a UV-vis spectrophotometer (UV-1800, Shimadzu, Japan). The electronic spectra of the reaction medium were recorded after 10 min mixing of the extract and $\mathrm{AgNO}_{3}$ solution. Before analysis, a small amount of the aliquot was diluted with deionized water. The range of wavelengths scanned was from 300 to $700 \mathrm{~nm}$.

2.8.2 Fourier transform infrared (FTIR) spectroscopy: For the conformation of the stabilizing agent on nanoparticles, FTIR spectral measurements were carried out on a Thermo Nicollet FTIR spectrometer (AVATAR model 5700, USA) using the KBr pellet method within a fixed spectral range (i.e. $4000-400 \mathrm{~cm}^{-1}$ ).

2.8.3 Thermogravimetric analysis (TGA): To study the thermal behavior of prepared AgNPs, TGA was carried out. TGA SII Exstar 6000, TG/DTA6300 thermal analyzer (Perkin-Elmer Life and Analytical Sciences, USA) was used at a rate of $10^{\circ} \mathrm{C} / \mathrm{min}$ in nitrogen atmosphere from $27^{\circ} \mathrm{C}$ to $900^{\circ} \mathrm{C}$.

2.8.4 Field-emission scanning electron microscopy (FE-SEM)/ energy-dispersive X-ray spectroscopy (EDX) analyses: The morphology, structure, and size of the synthesized AgNPs were recorded on an FE-SEM instrument (JSM-7600F, JEOL, Japan). Elemental analysis was investigated using Oxford-EDS system (JSM7600F, JEOL).
2.8.5 X-ray diffraction (XRD) analysis: For the determination of average crystallite size and shape, the prepared AgNP sample was analyzed using a JDX-3532 JEOL XRD ( $40 \mathrm{kV}, 30 \mathrm{~mA}$, monochromatic) using a CuK $\alpha$ source $(1.5418 \AA$ ). The scanning range used for the sample analysis was $20^{\circ} \leq 2 \theta \geq 70^{\circ}$ at a scanning rate of $0.5 \mathrm{~s} / \mathrm{step}$ and a step size of $0.05^{\circ}$. Scherrer equation was for the calculation of the average size of the nanoparticles:

$$
L=k \lambda \beta 1 / 2 \cos \theta
$$

where $\theta$ is the Bragg's angle, $\lambda$ is the wavelength (1.5418 $\AA$ ) of the $\mathrm{X}$-rays used, $\beta 1 / 2$ is the full peak width at half-maximum (FWHM) of the diffraction profile in radians on a $2 \theta$ scale, and $k$ constant is equal to unity related to both crystal shape and the way $\theta$ is defined.

\section{Results and discussion}

AgNPs were synthesized by treating $85 \mathrm{ml}$ solution of $3 \mathrm{mM} \mathrm{AgNO}_{3}$ with $15 \mathrm{ml} \mathrm{M}$. polymorpha leaf extract. On mixing the extract with the $\mathrm{AgNO}_{3}$ solution, a change in the color from light yellow to dark brown was observed by visual observation (Figure 1), and the change confirmed the reduction of $\mathrm{Ag}^{+}$to metallic silver.

\subsection{Mechanism of the reduction of $\mathrm{AgNO}_{3}$ to AgNPs}

The major phytoconstituents present in M. polymorpha are phenolic and flavonoid contents (i.e. gallic acid, polyphenols, pyrogallol, caffeic acid, and salicylic acid) [15]. A possible mechanism of the reduction of $\mathrm{Ag}^{+}$to AgNPs is proposed and presented in Scheme $1 . \mathrm{Ag}^{+}$may form an

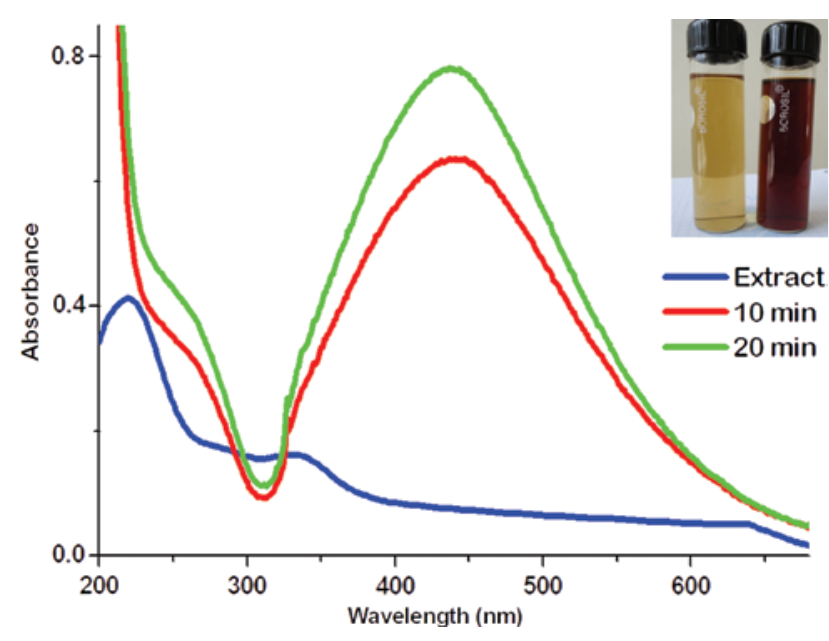

Figure 1: UV-vis spectra of $M$. polymorpha leaf extract and synthesized AgNPs. Experimental conditions: $85 \mathrm{ml}$ of $5 \mathrm{mM} \mathrm{AgNO}_{3}$ solution $+15 \mathrm{ml}$ of the $M$. polymorpha extract. Inset shows the change in color after $30 \mathrm{~min}$ and the formation of AgNPs. 


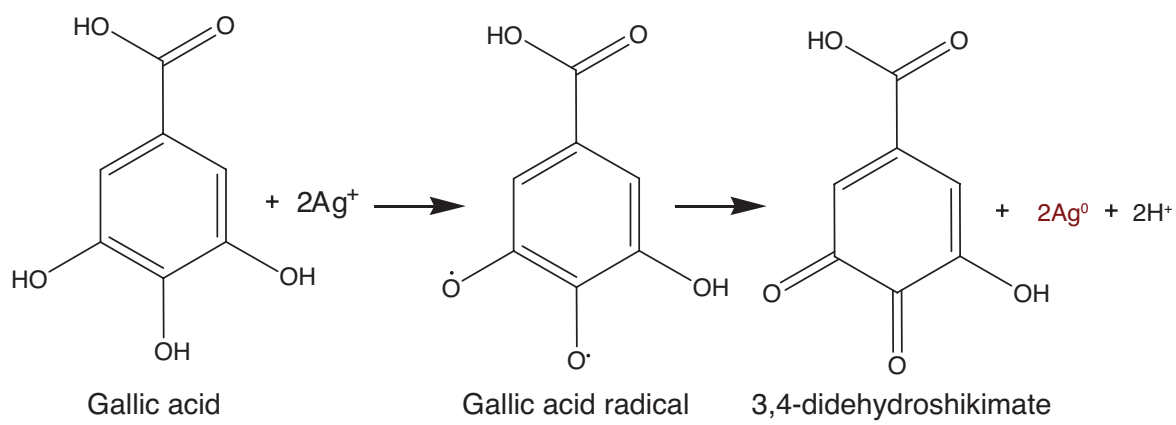

Scheme 1: Mechanism involved in the formation of AgNPs.

intermediate complex with phenolic moieties of gallic acid followed by oxidation to quinone (3,4-didehydroshikimate) with the consequential process of the conversion of $\mathrm{Ag}^{+}$to AgNPs [6].

\subsection{UV-vis analysis of AgNPs}

To investigate the bioreduction of silver ions to silver metal, the sample was monitored using a UV-vis spectrophotometer. The UV spectrum of the M. polymorpha leaf extract (Figure 1) showed an absorption band at $\lambda_{\max } 339 \mathrm{~nm}$ (bond I), which is characterized as the transition localized within the ring of the cinnamoyl system, whereas the other band at $225 \mathrm{~nm}$ (bond II) is characterized as the absorbance of the benzoyl-related system. These bands relate to $\pi \rightarrow \pi^{\star}$ transition, which demonstrate the presence of polyphenolics and antioxidant-like compounds in the extract [18, 19]. Figure 1 displays the UV-vis absorbance spectra of the synthesized AgNPs in the range of $200-700 \mathrm{~nm}$. The intensity of the color change increased with an increase in the incubation period of nanoparticles, which is attributable to the increase in the number of AgNPs formed in the reaction mixture. The characteristic surface plasmon resonance (SPR) peak of colloidal silver appeared at $430 \mathrm{~nm}[20,21]$. The fact that the AgNP band remained around $430 \mathrm{~nm}$ showed that the particles were well dispersed without aggregation in aqueous medium [22]. It has been reported in the literature that the absorption of AgNPs depends on the shape and size, so the formation of the shoulder at longer wavelength suggests that different sizes and shapes of particles were formed during the synthesis of AgNPs.

\subsubsection{FTIR analysis of AgNPs}

For the identification of stabilizing as well as reducing agents, FTIR analysis of the biosynthesized AgNPs was performed as FTIR spectroscopy is an important tool for the identification of the functional groups of biomolecules present in the plant extract. The major IR bands observed in the FTIR spectrum of the extract were 3278, 2915, 1640, $1519,1423,1269,1064,832$, and $628 \mathrm{~cm}^{-1}$ as shown in Figure 2. The broad band observed $3278 \mathrm{~cm}^{-1}$ is the characteristic of the hydroxyl functional group of phenolic compounds and various carbonyl groups of the M. polymorpha leaf extract. The band at $2915 \mathrm{~cm}^{-1}$ was assigned to the stretching vibration mode of methylene groups (alkyl C-H group), whereas the stretching vibration bands at 1650 and $1519 \mathrm{~cm}^{-1}$ with bending vibration at $628 \mathrm{~cm}^{-1}$ were the characteristic of the amide I and II linkages of amino acids and proteins present in the extract. The band $1423 \mathrm{~cm}^{-1}$ corresponds to the $\mathrm{C}=\mathrm{C}$ stretching vibration of aromatic rings. The band at $1269 \mathrm{~cm}^{-1}$ could be assigned to the $\mathrm{C}-\mathrm{O}$ stretching vibration of carboxylic acids. The peak at $1064 \mathrm{~cm}^{-1}$ may be due to the $\mathrm{C}-\mathrm{N}$ stretching vibration of aliphatic amines. The weak band at $832 \mathrm{~cm}^{-1}$ indicates the presence of stretching vibration of $\mathrm{N}-\mathrm{O}$ species [2325]. These bands correspond to the $-\mathrm{O}-\mathrm{H},-\mathrm{C}-\mathrm{N},-\mathrm{C}=\mathrm{N}$, $-\mathrm{C}-\mathrm{H},-\mathrm{C}-\mathrm{O}, \mathrm{C}-\mathrm{O}-\mathrm{H}, \mathrm{C}=\mathrm{C}$, and $\mathrm{N}-\mathrm{O}$ functional groups,

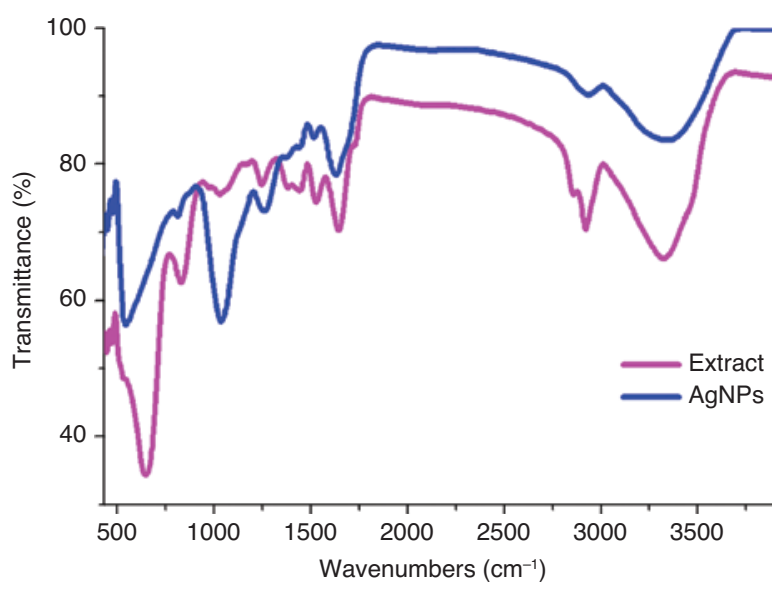

Figure 2: FTIR spectra of $M$. polymorpha leaf extract and synthesized AgNPs. 
which are derived from the water-soluble compounds such as flavonoids, amino acids, and polyphenols that are present in the M. polymorpha leaf extract.

The major IR bands observed in the spectrum of AgNPs were 3297, 2889, 1608, 1230, 1014, and $529 \mathrm{~cm}^{-1}$, as shown in Figure 2. In the case of AgNPs, the characteristic peaks of the plant extract appeared in FTIR along with a new peak at $529 \mathrm{~cm}^{-1}$, which corresponds to the metal-oxygen bond representing AgNPs. Hence, it can be concluded that these biomolecules apart from the reduction process could possibly form a covering layer that stabilize AgNPs (i.e. capping agent) and thereby stabilize AgNPs and prevent them from agglomeration or aggregation. The above results suggest that the leaf of the plant contained proteins, alkyl, amino, carbonyl, and hydroxyl functionalities, which have a noticeable affinity to the metal that probably facilitates metal ion reduction as well as stabilization to prevent the agglomeration of the nanoparticles.

\subsubsection{TGA study of AgNPs}

To check the thermal behavior of synthesized nanoparticles under high temperature, the purified AgNPs were subjected to TGA. Figure 3 shows the thermogram of the M. polymorpha extract reduced AgNPs. Three different endothermic peaks $\left(78^{\circ} \mathrm{C}, 270^{\circ} \mathrm{C}\right.$, and $\left.530^{\circ} \mathrm{C}\right)$ are due to the evaporation of physically adsorbed water molecules, decomposition of the proteins, and biomass that covers the particle surface. The initial weight loss was observed up to $133^{\circ} \mathrm{C}$ and attributed to the water molecules present in the AgNPs. A steady weight loss was observed until $690^{\circ} \mathrm{C}$. We believe that this weight loss amounting to

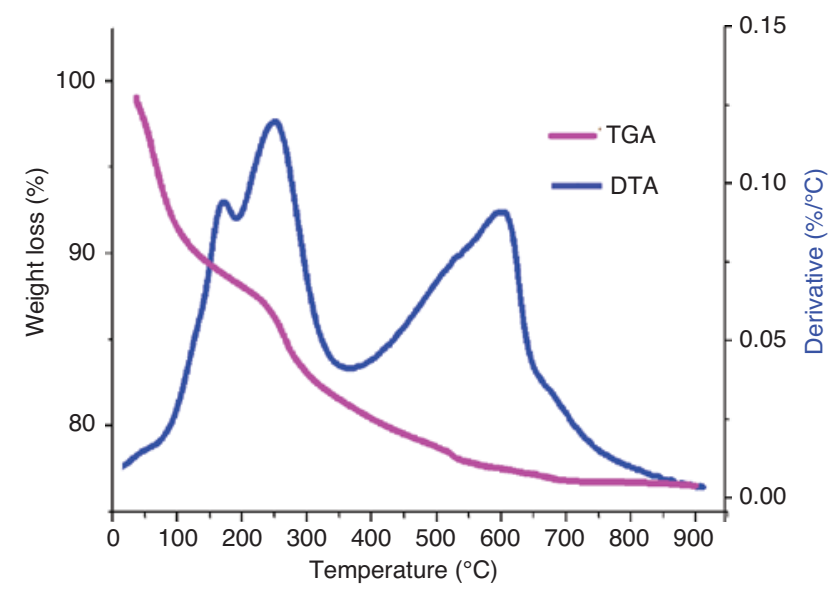

Figure 3: TGA/DTA thermogram of the synthesized AgNPs.
$23.48 \%$ of the sample weight is due to the desorption of bioorganic components present in the AgNPs. In the end, residual silver was about $76.52 \%$. A phase transition was seen at a temperature of $900^{\circ} \mathrm{C}$, close to the melting point of silver metal.

\subsubsection{FE-SEM/EDX profile of AgNPs}

The surface morphology of synthesized AgNPs was investigated using FE-SEM. The micrograph showed that the biosynthesized AgNPs were predominantly spherical in shape as shown in Figure 4A and B. The crystallite size thus obtained from FE-SEM of nanoparticles was found within the range of $25-33 \mathrm{~nm}$ (mean: $\sim 29 \pm 4 \mathrm{~nm}$ ), which was ably supported by XRD pattern too. The particle size distribution histogram obtained from the AgNPs showed that the particle size range was $15-50 \mathrm{~nm}$. The particle size distribution histogram in Figure 4C showed that the average nanoparticle size was between 25 and $33 \mathrm{~nm}$. These results indicated that AgNPs synthesized using M. polymorpha leaf produced crystalline nanoparticles. EDX analysis furnished additional evidence for the confirmation of AgNP synthesis by M. polymorpha as shown in Figure 4D. A strong characteristic signal peak for metallic silver was observed at $3 \mathrm{keV}$ due to SPR [26, 27]. The spectrum also showed weak signals for oxygen and carbon, which may have originated from biomolecules that stabilize the surface of AgNPs. EDX data proved the chemical purity and synthesis of AgNPs.

\subsubsection{XRD pattern of synthesized AgNPs}

The XRD pattern of biogenic AgNPs is shown in Figure 5. It can be seen that there are four intense peaks in the whole spectrum of $2 \theta$ (15-70). Three distinct diffraction peaks at the $2 \theta$ positions of $38.05^{\circ}, 46.3^{\circ}$, and $65.65^{\circ}$ correspond to Bragg peaks of (111), (200), and (220), which corresponded to the face-centered cubic of AgNPs [28]. The low intensity and broadness of the peaks revealed the small crystallite size of the AgNPs. From XRD, the average crystallite size of the nanoparticles was calculated using the Scherrer formula, where the average particle size was calculated by the FWHM of the most intense peak. The average crystallite size of nanoparticles was thus found to be $\sim 30 \pm 5 \mathrm{~nm}$, which was ably supported by FE-SEM analysis too. These results indicated that AgNPs synthesized using $M$. polymorpha leaf produced crystallite in nanosize. Some unidentified crystalline peaks $\left(18.3^{\circ}, 20.35^{\circ}, 24.75^{\circ}\right.$, $30.9^{\circ}, 43.45^{\circ}$, and $57.45^{\circ}$ ) were also seen in the literature 

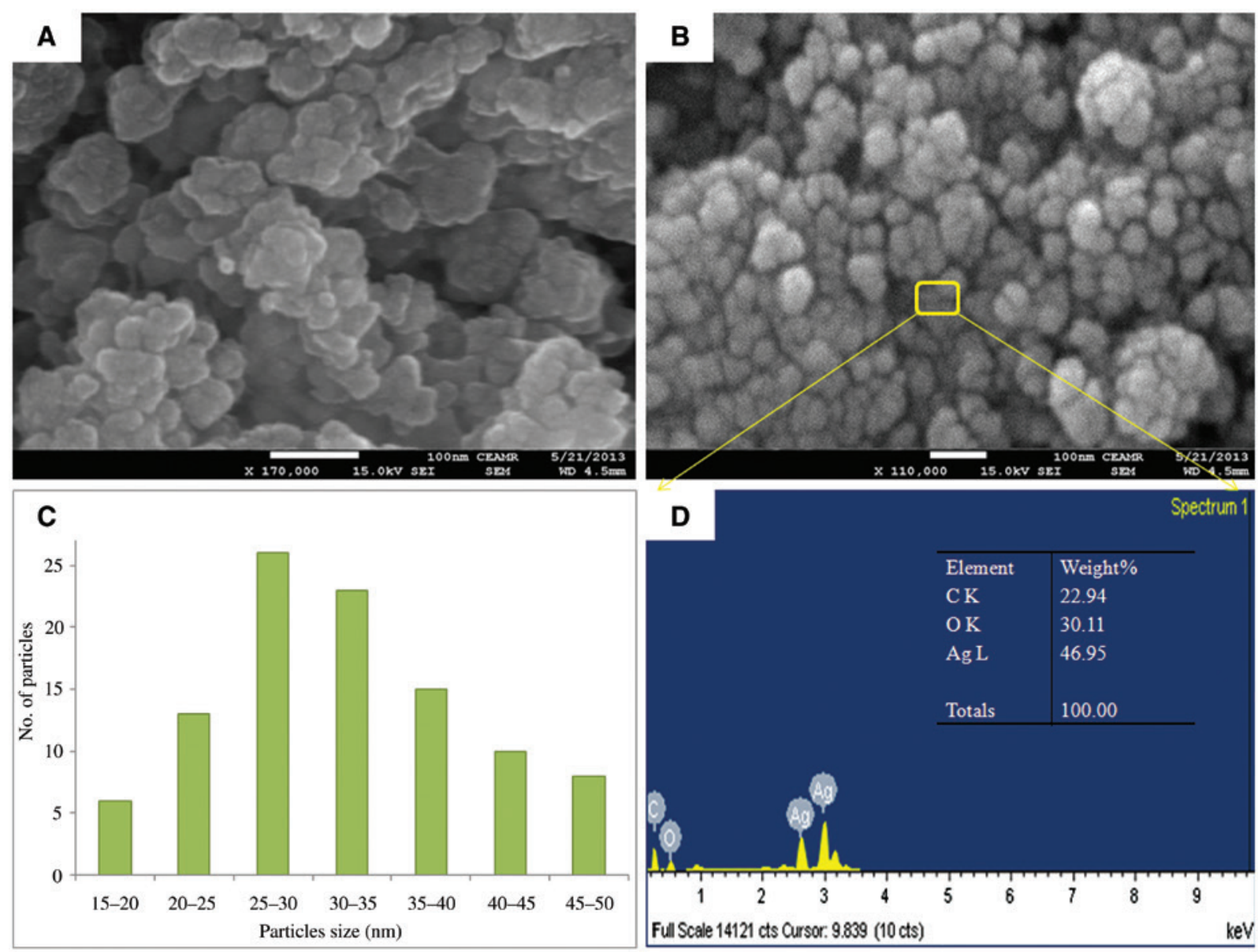

Figure 4: (A and B) High- to low-magnification FE-SEM images, (C) particle size distribution, and (D) EDX investigation of AgNPs.

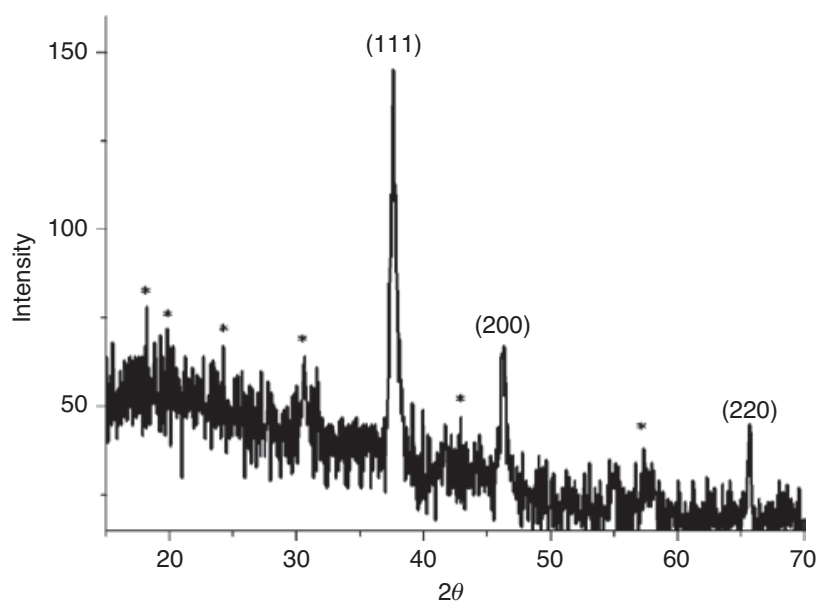

Figure 5: Powder XRD pattern of AgNPs. *Indicates unassigned peaks.

in which the XRD pattern included the relevant $2 \theta$ range. These unassigned peaks ${ }^{\star}{ }^{*}$ are due to the crystallization of bioorganic molecules that act as capping agents that are present in the extract [29].

\subsection{Antibacterial activity of AgNPs}

\subsubsection{MIC of AgNPs}

Synthesized AgNPs displayed an appreciable antibacterial effect. MIC was the lowest concentration at which no visible growth of pathogens was observed (Figure 6). The MIC observed at $10 \mu \mathrm{g} / \mathrm{ml}$ for AgNPs and at $20 \mu \mathrm{g} / \mathrm{ml}$ for $\mathrm{AgNO}_{3}$ was recorded against $E$. coli and $S$. aureus. Lower MIC of AgNPs than $\mathrm{AgNO}_{3}$ may be due to the nanoscale nature of the biologically synthesized material [30, 31]. On the contrary, the $M$. polymorpha extract was unable to exhibit any activity; hence, no value of MIC was recorded.

\subsubsection{Changes in membrane permeability of bacterial cells}

To ascertain the interaction of AgNPs and bacterial surfaces, a membrane permeability test was performed. About $10 \mathrm{ml}$ of log-phase cultures of E. coli and S. aureus 

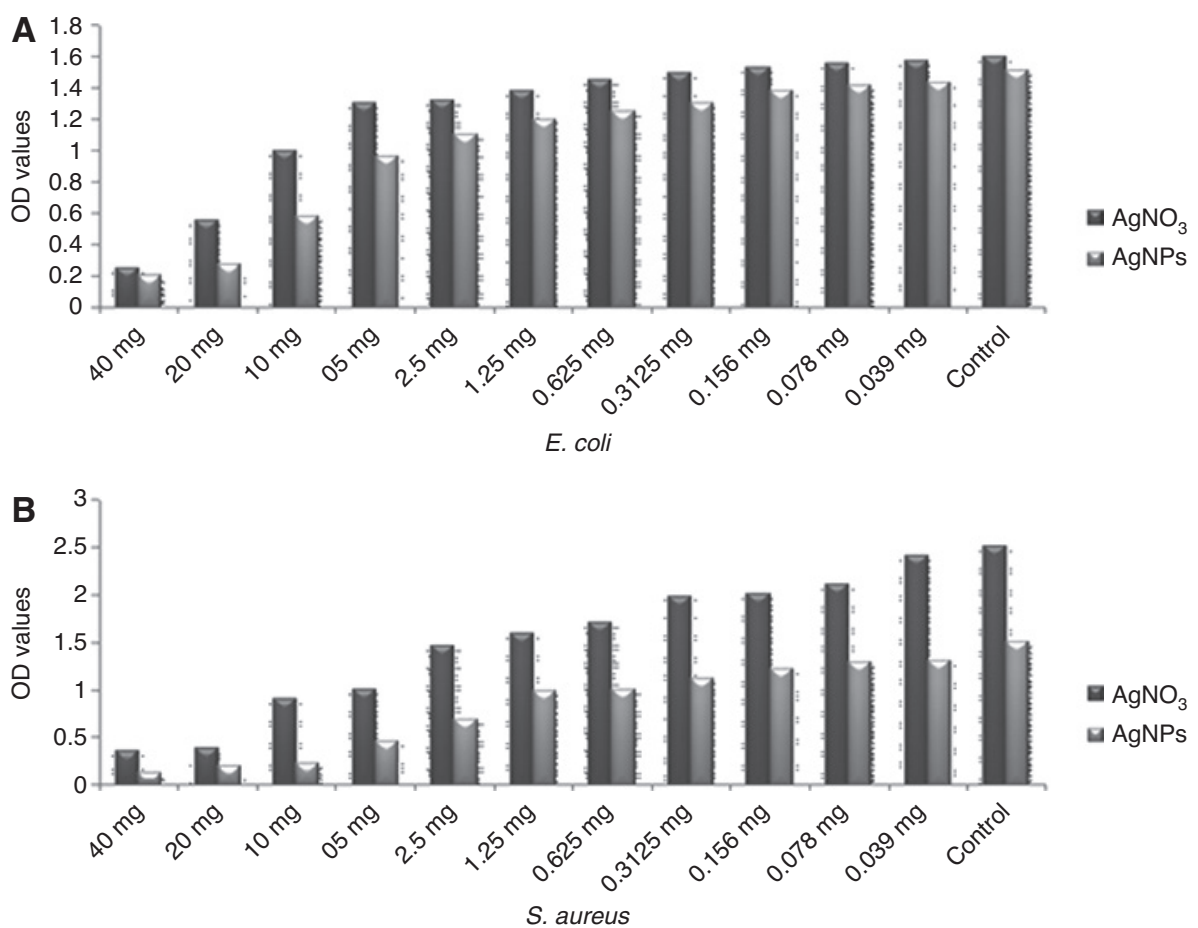

Figure 6: $\mathrm{MICS}$ of $\mathrm{AgNO}_{3}$ and AgNPs on (A) E. coli and (B) S. aureus.

exposed to $100 \mathrm{ppb}$ AgNPs showed significant conductivity of $268,288 \mu \mathrm{S} / \mathrm{cm}$ compared to $\mathrm{AgNO}_{3}$-treated broth $(169,199 \mu \mathrm{S} / \mathrm{cm}$; Figure 7$)$. Significant conductivity may be attributed to the serious damage of the cell membrane structure caused by AgNPs due to the smaller size of the material, which led to an increase in membrane permeability/cell destruction. The mechanism of antibacterial action of AgNPs is still not well understood to date; however, this action involves the formation of "pits" in the

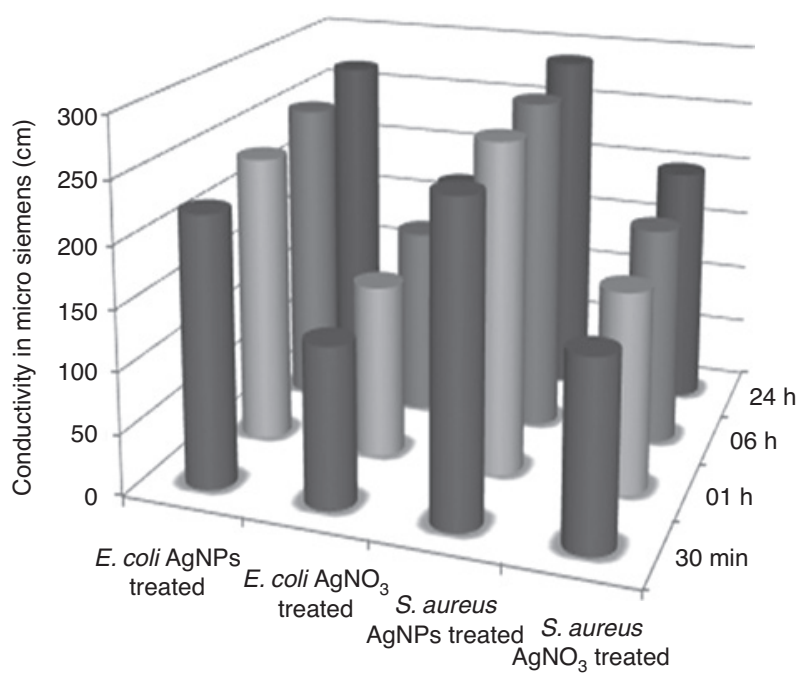

Figure 7: Membrane permeability study of E. coli and S. aureus. bacterial cell wall, which ultimately led to the increased membrane permeability and cell death [32]. The literature also reported an antibacterial action of the $\mathrm{Ag}^{+}$primarily caused by their interaction with the cytoplasm; briefly, the $\mathrm{Ag}^{+}$appeared to penetrate through ion channels through the cell membranes. The denaturing of ribosome resulted in the suppression of the enzymes expression, thereby disrupting the cell [33]. The increased conductance of the cell culture treated with AgNPs could be easily attributed to the dissolution of the cellular contents by the disruption of the cell membrane structures with the loss of membrane permeability.

\subsection{Antioxidant activity of AgNPs}

To elevate the antioxidant activity of M. polymorpha synthesized AgNPs, the RSA of the aqueous leaf extract of M. polymorpha and AgNPs was evaluated using DPPH assay. Figure 8 shows that the aqueous leaf extract of M. polymorpha and AgNPs demonstrate excellent DPPH radical inhibitory activity, indicating a source for antioxidants. For RSA, different concentrations (10, 50, and $100 \mu \mathrm{g} / \mathrm{ml})$ of the aqueous leaf extract of M. polymorpha, AgNPs, and positive control ascorbic acid were used to react with equimolar concentrations of $50 \mu \mathrm{M}$ DPPH solution. Here, we used $50 \mu \mathrm{M}$ concentration of DPPH in consonance with the 


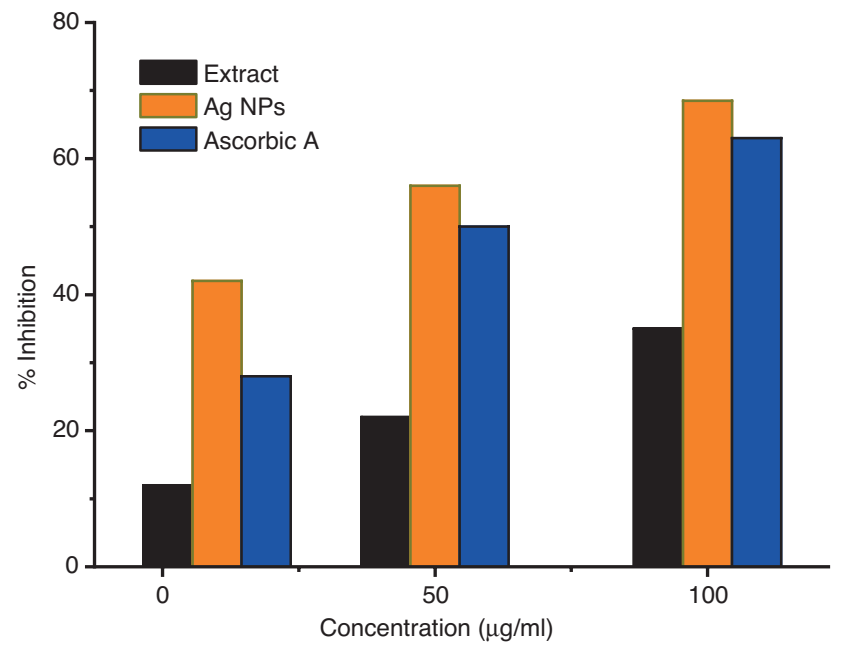

Figure 8: DPPH RSA of $M$. polymorpha leaf extract and synthesized AgNPs.

accuracy in measurements of the spectrophotometer [34]. The antioxidant activity of the M. polymorpha leaf extract, AgNPs, and ascorbic acid was found to increase in a dosedependent manner. Compared to the positive control ascorbic acid, the AgNPs exhibited a stronger RSA. Most importantly, M. polymorpha-mediated AgNPs exhibited an excellent antioxidant activity with $68.5 \%$ DPPH scavenging activity. M. polymorpha-mediated AgNPs showed higher DPPH scavenging activity then previously reported Asphodelus aestivus-mediated AgNPs [35]. However, the aqueous leaf extract exhibited lower antioxidant activity than previously reported aqueous extract of Salicornia brachiata [36]. Silver colloids exhibited a higher antioxidant activity than M. polymorpha leaf extract at all tested concentrations. It is evident that the resulting excellent antioxidant activity of the AgNPs is due not only to the stabilizing agents (biomolecules present in the M. polymorpha extract) but also to the elemental silver and can be used for the treatment of several oxidative stress diseases and the control of various human and veterinary infections.

\subsection{Catalytic reduction of MO dye}

The discharge of dye effluents to the aquatic environment from the textile industry is the main source of water
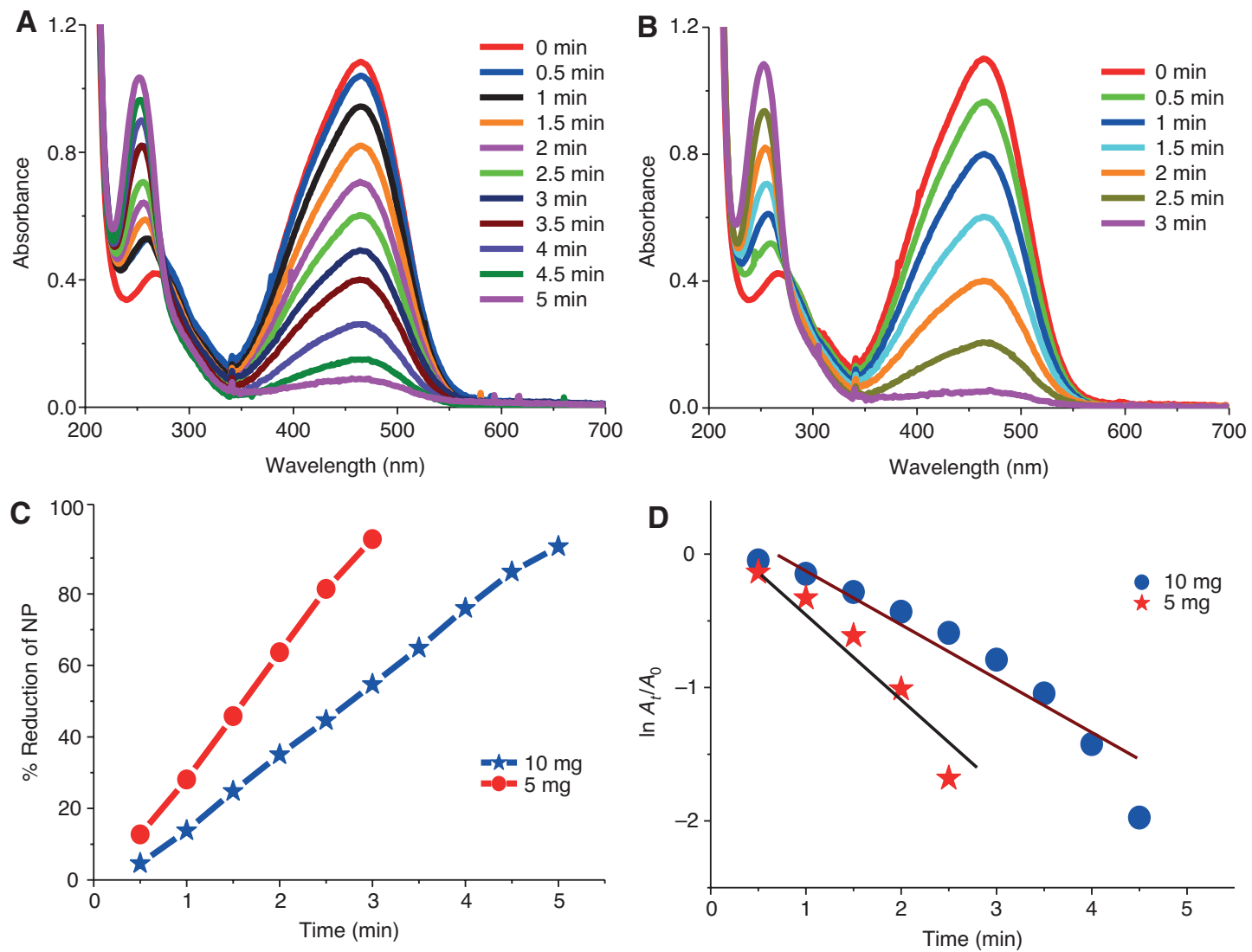

Figure 9: UV-vis spectra of (A) MO reduction by $\mathrm{NaBH}_{4}$ and $5 \mathrm{mg}$ AgNPs and (B) $10 \mathrm{mg} \mathrm{AgNPs,} \mathrm{(C)} \mathrm{comparative} \mathrm{percent} \mathrm{reduction} \mathrm{of} \mathrm{MO}$ dye, and (D) plot of $\ln \left(A_{t} / A_{0}\right)$ versus time for MO. Experimental conditions: $3 \mathrm{ml}$ of aqueous $\mathrm{MO}$ solution $+0.5 \mathrm{ml}$ of $0.5 \mathrm{M} \mathrm{NaBH}{ }_{4} \mathrm{solution}_{+} \mathrm{AgNP}$ catalyst (5 and $10 \mathrm{mg}$ ). 
pollution. MO is one of the toxic pollutants of wastewater and poses a potential threat to the environment. MO is mostly used in textile industries and as an indicator in the laboratory. The presence of the azo group and the low biodegradability of MO make it an important concern for environmental hazards [37]. Thus, the treatment of MO is highly desirable.

$\mathrm{MO}$ is a persistent molecule and the reduction of $\mathrm{MO}$ by $\mathrm{NaBH}_{4}$ in the absence of any suitable catalyst is kinetically unfavorable but thermodynamically favorable. It has been reported in the literature that, in the absence of a catalyst, the peak intensity at $465 \mathrm{~nm}$ of MO remains constant even for several hours [38, 39].

For the reduction, here we first incorporated $3 \mathrm{ml}$ of $0.02 \mathrm{~mm} \mathrm{MO}$ and $0.5 \mathrm{ml}$ of $\mathrm{NaBH}_{4}$ solution. The aqueous solution of $\mathrm{MO}$ gives a strong absorption peak in the visible region at $465 \mathrm{~nm}$ and a weak absorption at $282 \mathrm{~nm}$. The UV-vis spectra of the reaction were recorded constantly with a time interval of $30 \mathrm{~s}$ as shown in Figure 9A and B. The decrease in $\lambda_{\max }$ at both 282 and $465 \mathrm{~nm}$ started after the insertion of $5 \mathrm{mg}$ dried AgNPs. The peak intensity at $465 \mathrm{~nm}$ gradually decreased with time, whereas the intensity of the peak at $282 \mathrm{~nm}$ slightly shifted toward the lower wavelength with increase in absorption and reached $251 \mathrm{~nm}$ as shown in Figure 9A and B. The decreased in the peak intensity at $465 \mathrm{~nm}$ with gradual disappearance after the addition of AgNPs was due to the beginning of MO reduction, which were completed in short times of 3 and $5 \mathrm{~min}$ for 10 and $5 \mathrm{mg}$ AgNPs, respectively. It has been reported in the literature that $\mathrm{NaBH}_{4}$ in the presence of an active catalyst reduces the MO molecule at the azo site $(-\mathrm{N}=\mathrm{N}-)$ by generating low molecular amino compounds. Thus, the new peak appearance with an increase in intensity at $251 \mathrm{~nm}$ was considered from the compound of the $-\mathrm{NH}_{2}$ group generated during the reduction of $\mathrm{MO}$ [38].

The reduction of $\mathrm{MO}$ by $\mathrm{NaBH}_{4}$ follows the "pseudofirst-order" kinetic. Thus, the rate constants $\left(k_{\text {app }}\right)$ calculated from the slope at room temperature for 5 and $10 \mathrm{mg}$ AgNPs were $6.8 \times 10^{-3}$ and $5.8 \times 10^{-3} \mathrm{~s}^{-1}$, respectively (Figure 9D). The percent reduction of MO by AgNPs was calculated using Equation (3) from their UV-vis spectra:

$$
\% \text { Reduction }=100-\left(A_{t} \times 100 / A_{0}\right)
$$

where $A_{0}$ is the initial absorbance $\left(\lambda_{\max }\right.$ at $\left.465 \mathrm{~nm}\right)$ and $A_{t}$ is the absorbance at the reaction time $t$. The prepared nanoparticles showed an excellent MO reduction, and a $97 \%$ reduction took place in 5 and $3 \mathrm{~min}$ for 5 and $10 \mathrm{mg}$ AgNPs, respectively (Figure 9C). Thus, the reduction of MO by AgNPs is very high compared to the previously reported study in which about $80 \%$ degradation of MO took $45 \mathrm{~min}$ using Mussaenda erythrophylla leaf-mediated AgNPs [40]. This indicates that our prepared AgNPs showed outstanding catalytic reduction activity over MO dye.

\section{Conclusions}

The present study is the first ever report that demonstrated the role of $M$. polymorpha as a natural low-cost biological reducing agent for AgNP synthesis at room temperature. This is a simple, ecofriendly, and efficient one-step route for the preparation of AgNPs at room temperature without using any toxic reducing and capping agents. XRD data proved that the mean size of the synthesized AgNPs was $\sim 30 \pm 5 \mathrm{~nm}$, which was ably supported by FE-SEM analysis too. As evidenced by antibacterial and catalytic degradation activities, AgNPs were more active than the precursors. Overall, the presented strategy is a simple and cost-efficient way of nanomedicine for the control of azo dye pollutions, treatment of several oxidative stress diseases, and control of various human and veterinary infections.

Acknowledgments: The authors are highly grateful for the Center of Excellence for Advance Materials Research and Chemistry Department, King Abdulaziz University, Jeddah, Saudi Arabia, and the Department of Chemistry, Kohat University of Science and Technology, Kohat, Pakistan.

Conflict of interest statement: The authors declare no conflict of interests.

\section{References}

[1] Niraimathi K, Sudha V, Lavanya R, Brindha P. Colloids Surf. B 2013, 102, 288-291.

[2] Kaviya S, Santhanalakshmi J, Viswanathan B. Mater. Lett. 2012, 67, 64-66.

[3] Gopinath V, MubarakAli D, Priyadarshini S, Priyadharsshini NM, Thajuddin N, Velusamy P. Colloids Surf. B 2012, 96, 69-74.

[4] Narayanan KB, Sakthivel N. Mater. Charact. 2010, 61, 1232-1238.

[5] Gul S, Ismail M, Khan MI, Khan SB, Asiri AM, Rahman IU, Khan MA, Kamboh MA. Asian Pac. J. Trop. Dis. 2016, 6, 311-316.

[6] Ismail M, Gul S, Khan MA, Khan M. Rev. Nanosci. Nanotechnol. 2016, 5, 119-135.

[7] Priyadarshini S, Gopinath V, Priyadharsshini NM, MubarakAli D, Velusamy P. Colloids Surf. B 2013, 102, 232-237.

[8] Jeyaraj M, Rajesh M, Arun R, MubarakAli D, Sathishkumar G, Sivanandhan G, Dev GK, Manickavasagam M, Premkumar K, Thajuddin N. Colloids Surf. B 2013, 102, 708-717. 
[9] Dwivedi AD, Dubey SP, Sillanpää M, Liimatainen H, Suopajärvi T, Niinimäki J, Kwon Y-N, Lee C. Int. J. Biol. Macromol. 2015, 76, 109-118.

[10] Dar MA, Ingle A, Rai M. Nanomed. Nanotechnol. Biol. Med. 2013, 9, 105-110.

[11] Kansal SK, Lamba R, Mehta S, Umar A. Mater. Lett. 2013, 106, 385-389.

[12] Kamal T, Khan SB, Asiri AM. Cellulose 2016, 23, 1911-1923.

[13] Ismail M, Khan MI, Khan SB, Khan MA, Akhtar K, Asiri AM. J. Mol. Liq. 2018, 260, 78-91.

[14] Ghule LA, Patil AA, Sapnar KB, Dhole SD, Garadkar KM. Toxicol. Environ. Chem. 2011, 93, 623-634.

[15] Arabhosseini A, Huisman W, Müller J. Ind. Crops Prod. 2011, 34, 1550-1555.

[16] Edison TJI, Sethuraman M. Process Biochem. 2012, 47, 1351-1357.

[17] Ramamurthy CH, Padma M, Mariya Samadanam ID, Mareeswaran R, Suyavaran A, Kumar MS, Premkumar K, Thirunavukkarasu C. Colloids Surf. B 2013, 102, 808-815.

[18] Nasrollahzadeh M, Maham M, Rostami-Vartooni A, Bagherzadeh M, Sajadi SM. RSC Adv. 2015, 5, 64769-64780.

[19] Nasrollahzadeh M, Sajadi SM, Khalaj M. RSC Adv. 2014, 4, 47313-47318.

[20] Chang G, Luo Y, Lu W, Liao F, Sun X. J. Nano. Res. 2011, 13, 2689-2695.

[21] Qin X, Luo Y, Lu W, Chang G, Asiri AM, Al-Youbi AO, Sun X. Electrochim. Acta 2012, 79, 46-51.

[22] Bindhu M, Umadevi M. Spectrochim. Acta A 2013, 101, 184-190.

[23] Antony JJ, Nivedheetha M, Siva D, Pradeepha G, Kokilavani P, Kalaiselvi S, Sankarganesh A, Balasundaram A, Masilamani V, Achiraman S. Colloids Surf. B 2013, 109, 20-24.
[24] Kannan RRR, Stirk WA, Van Staden J. S. Afr. J. Bot. 2013, 86, 1-4.

[25] Nasrollahzadeh M, Sajadi SM. RSC Adv. 2015, 5, 46240-46246.

[26] Kamal T, Ahmad I, Khan SB, Asiri AM. Carbohydr. Polym. 2017, 157, 294-302.

[27] Lu W, Liao F, Luo Y, Chang G, Sun X. Electrochim. Acta 2011, 56, 2295-2298.

[28] Banala RR, Nagati VB, Karnati PR. Saudi J. Biol. Sci. 2015, 22, 637-644.

[29] Krishnaraj C, Jagan EG, Rajasekar S, Selvakumar P, Kalaichelvan PT, Mohan N. Colloids Surf. B 2010, 76, 50-56.

[30] Oves M, Arshad M, Khan MS, Ahmed AS, Azam A, Ismail IMI. J. Saudi Chem. Soc. 2015, 19, 581-588.

[31] Kumar DA, Palanichamy V, Roopan SM. Spectrochim. Acta A 2014, 127, 168-171.

[32] Roopan SM, Rohit, Madhumitha G, Rahuman AA, Kamaraj C, Bharathi A, Surendra TV. Ind. Crops Prod. 2013, 43, 631-635.

[33] Sathiya CK, Akilandeswari S. Spectrochim. Acta A 2014, 128, 337-341.

[34] Sharma OP, Bhat TK. Food Chem. 2009, 113, 1202-1205.

[35] Fafal T, Taştan P, Tüzün BS, Ozyazici M, Kivcak B. S. Afr. J. Bot. 2017, 112, 346-353.

[36] Seralathan J, Stevenson P, Subramaniam S, Raghavan R, Pemaiah B, Sivasubramanian A, Veerappan A. Spectrochim. Acta A 2014, 118, 349-355.

[37] Munagapati VS, Yarramuthi V, Kim D-S. J. Mol. Liq. 2017, 240, 329-339.

[38] Khan MM, Lee J, Cho MH. J. Ind. Eng. Chem. 2014, 20, 1584-1590.

[39] Ali F, Khan SB, Kamal T, Anwar Y, Alamry KA, Asiri AM. Carbohydr. Polym. 2017, 173, 676-689.

[40] Varadavenkatesan T, Selvaraj R, Vinayagam R. J. Mol. Liq. 2016, 221, 1063-1070. 